\title{
The description and phylogenetic position of a new nanhsiungchelyid turtle from the Late Cretaceous of Mongolia
}

\author{
Vladimir B. Sukhanov, Igor G. Danilov, and Elena V. Syromyatnikova \\ Acta Palaeontologica Polonica 53 (4), 2008: 601-614 doi:http://dx.doi.org/10.4202/app.2008.0405
}

This paper describes a new nanhsiungchelyid turtle, Kharakhutulia kalandadzei gen. et sp. nov., based on two partial

shells and additional shell fragments from the lower part of the Bainshire Formation

(Upper Cretaceous, Cenomanian-lower Turonian) of the Khara Khutul locality of Eastern

Mongolia. Our phylogenetic analysis places Kharakhutulia kalandadzei as the most

basal member of the Nanhsiungchelyidae and suggests new relationships within this

group. Previously reported nanhsiungchelyid specimens from the Khara Khutul are reassigned to Nanhsiungchelyidae indet. and Hanbogdemys sp. indet. Thus the Khara Khutul includes at least two valid taxa of nanhsiungchelyids. Our analysis of the nanhsiungchelyid record in Asia shows that other localities have only a single representative of this clade, making Khara Khutul a unique site. The basal phylogenetic position of Kharakhutulia kalandadzei emphasizes the importance of the study of this and other Cenomanian-Turonian localities of Asia to better understand the basal diversification of the Nanhsiungchelyidae.

Key words: Kharakhutulia, Nanhsiungchelyidae, Cryptodira, Testudines, Upper Cretaceous, Mongolia.

Vladimir B. Sukhanov[sukhanovturtle@yandex.ru], Paleontological Institute, Russian Academy of Sciences, Profsoyuznaya 123, 117997, Moscow, Russia. Igor G. Danilov [dig@mail333.com], Department of Herpetology, Zoological Institute, Russian Academy of Sciences, Universitetskaya Emb. 1, 199034, St. Petersburg, Russia (corresponding author); Elena V. Syromyatnikova[aravir@ mail.ru], Laboratory of Theriology, Zoological Institute, Russian Academy of Sciences, Universitetskaya Emb. 1, 199034, St. Petersburg, Russia.

This is an open-access article distributed under the terms of the Creative Commons Attribution License (for details please see creativecommons.org), which permits unrestricted use, 
distribution, and reproduction in any medium, provided the original author and source are credited.

Fof Full text $(900.0 \mathrm{kB})$ 